Ann. Zootech., I975, 24 (2), 289-298.

\title{
ÉVOLUTION, AU COURS DE LA CROISSANCE, DES GARACTÉRISTIQUES DE LA CONSOMMATION D'ALIMENTS SOLIDE ET LIQUIDE DU LAPIN DOMESTIQUE NOURRI $A D$ LIBITUM
}

\author{
M. PRUD'HON, Michaelle CHÉRUBIN, J. GOUSSOPOULOS et Y. CARLES \\ Station de Physiologie animale, I. N. R. A., \\ E.N.S. A., 9 place Viala, \\ 34060 Montpellier Cedex
}

\section{RÉSUMÉ}

L'amplitude et la répartition des prises d'aliments solides et liquides de neuf lapins mâles Néo-Zélandais blancs ont été enregistrées graphiquement au cours des sixième, neuvième, douzième, quinzième et dix-huitième semaines. Le dispositif d'enregistrement était celui décrit par Prud'hon, Carles et Goussopoulos (i973).

Le comportement alimentaire du lapin en croissance est caractérisé par :

- l'absence de véritables repas mais une succession de prises d'aliments et d'eau d'amplitude et d'espacement très irréguliers ;

- une répartition nycthémérale de la consommation étroitement liée à l'allumage et l'extinction de la lumière :

- l'accentuation progressive du caractère nocturne des consommations.

Quantitativement les consommations d'aliment et d'eau croissent jusqu'à la quinzième semaine et diminuent à la dix-huitième. L'accroissement est dû :

— entre 6 et 9 semaines à l'augmentation d'amplitude des prises ;

- après neuf semaines, à un accroissement de la fréquence nocturne des prises, leur amplitude variant peu ; simultanément la fréquence des prises diurnes a tendance à diminuer. L'amplitude des prises diurnes et nocturnes est égale quel que soit l'âge. Le rapport eau/matière sèche augmente avec l'âge (de I,86 jusqu'à 2,22).

La répartition nycthémérale des consommations est caractérisée par :

- une période de faible consommation diurne, de courte durée à 6 semaines ( 3 heures) et de longue durée à $\mathbf{I} 8$ semaines ( 5 à 7 heures). En outre à 6 semaines existe une période de faible consommation nocturne qui disparaît chez les sujets plus âgés.

- une consommation intense d'aliment dans l'heure qui précède l'extinction de la lumière ; la consommation d'eau de boisson présente ce pic à 6 semaines mais non à 18 . A cet âge la consommation est maximale en fin de nuit. 


\section{INTRODUCTION}

Nous avons déjà souligné (PRUD'HON et al., I972) la nécessité d'acquérir une meilleure connaissance du comportement spontané de prise d'aliments solide et liquide si l'on désire contrôler le niveau d'ingestion des sujets en croissance. Nous avons décrit deux dispositifs d'enregistrement graphique continu (PRUD'HON et al., I972., Prud'hon, Carl,ks et Goussopoulos, I973) suffisamment précis et fidèles pour permettre la mesure de l'amplitude et de la répartition temporelle des repas chez le Lapin domestique. Les caractéristiques de la consommation de lapins adultes des deux sexes ont été précisées.

La présente étude est une analyse de l'évolution de la fréquence, de l'importance et de la répartition de la consommation d'eau et d'aliments au cours de la croissance du Lapin Néo-Zélandais, de l'âge de 5 à I 8 semaines.

\section{MATÉRIEL, E'T MÉTHODES}

\section{Dispositif d'enregistrement graphique}

Le dispositif d'enregistrement graphique a été décrit en détail (Prud'hon, Carles et Goussopoulos, 1973) rappelons ses principes.

L'eau de boisson est distribuée par un abreuvoir à clapet alimenté par un réservoir cylindrique maintenu sous pression par un piston étanche dont on enregistre le déplacement; le remplissage du réservoir se fait automatiquement lorsque le niveau s'abaisse au-dessous d'un seuil donné.

L'aliment solide, granulé, est distribué dans une trémie s'ouvrant sur la cage. La trémie est placée sur le plateau d'une balance qui supporte également un réservoir compensateur ; la consommation d'aliments entraîne l'envoi d'une quantité d'eau de poids égal dans le réservoir compensateur; le déplacement du piston assurant le remplissage est enregistré.

Afin d'éviter que les oscillations accidentelles de la trémie, provoquées par exemple par la prise d'appui du lapin sans consommation réelle, ne provoquent un remplissage intempestif du réservoir compensateur, un temporisateur permet de différer d'une minute la remise en équilibre.

Les trois cages équipées de ce dispositif sont situées dans une pièce, le dispositif d'enregistrement proprement dit dans la pièce contiguë afin de ne pas troubler les sujets expérimentaux lors du contrôle du bon fonctionnement de celui-ci. Les consommations d'eau et de granulé peuvent être lues à $0,25 \mathrm{~g}$ près, l'intervalle entre deux repas à deux minutes près. La recharge des trémies est effectuée une fois par semaine.

\section{Conditions expérimentales}

Les mesures ont porté sur 9 lapins mâles de race Néo-Zélandais blanc répartis en trois groupes de trois; ils étaient sevrés à 4 semaines, acclimatés aux conditions expérimentales durant une semaine puis enregistrés au cours des sixième, neuvième, douzième, quinzième et dix-huitième semaines. Entre deux périodes d'enregistrement ils étaient maintenus dans la salle de contrôle pendant qu'une autre série de trois lapins était contrôlée.

Pendant toute la période expérimentale la température du local était maintenue à $20 \pm \mathrm{I}^{\circ} \mathrm{C}$, l'éclairement d'une intensité lumineuse de I 2 lux au niveau des cages était assuré par des tubes fluorescents de type "Iumière du jour ». La durée d'éclairement était de I4 heures sur 24 heures, distribuée entre 7 heures et 21 heures.

L'aliment était un granulé du commerce dosant au minimum 15 p. Ioo de matières protéiques brutes et au maximum i3 p. Ioo de cellulose. 


\section{RÉSULTATS}

\section{A. - Caractéristiques générales de la consommation d'aliment et d'eau de boisson au cours de la croissance}

L'aspect général des diagrammes d'enregistrement des prises d'aliments ( ${ }^{1}$ ) et d'eau (fig. I) est très semblable à celui que nous avions déjà enregistré chez des sujets adultes :

Consommation d'aliment par prises successives, bien individualisées, séparées les unes des autres par des intervalles d'une durée très variable (de moins de 5 minutes à plus de 5 heures) ; consonmation d'eau présentant les mêmes caractéristiques sans qu'il n'y ait d'alternance régulière entre prise d'aliment et d'eau.

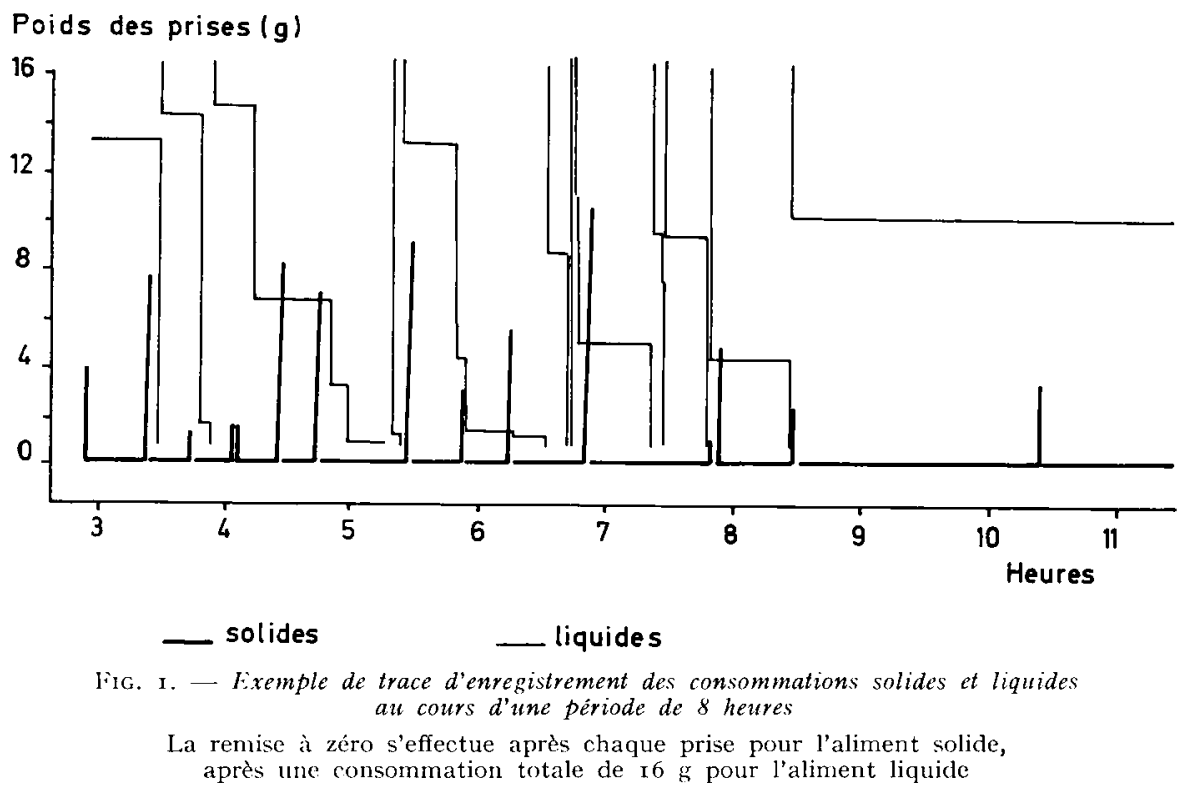

L'étude attentive des polygones de fréquences des intervalles entre prises (fig. 2), ne permet pas de définir de véritables périodes de repas séparées par des périodes de repos alimentaire. En effet, dans cette hypothèse on observerait deux types d'intervalles : des intervalles de courte durée correspondant aux repos entre les différentes prises d'un même repas et des intervalles de longue durée correspondant aux repos qui séparent les repas successifs ; la distribution serait bimodale ce qui n'est le cas ni de jour ni de nuit. Par contre, ainsi que cela apparaît sur la figure 3 qui correspond à la juxtaposition des consommations horaires journalières de l'un des lapins

(1) Sous utiliserons pour simplifier le terme aliment lorsqu'il s'agit de l'aliment solide. 
Frequence

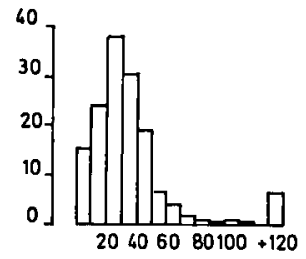

$20406080100+120$

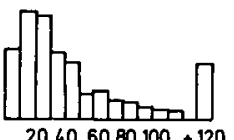

$20606080100+120$

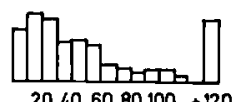

$20406080100+120$

Intervalles en minutes

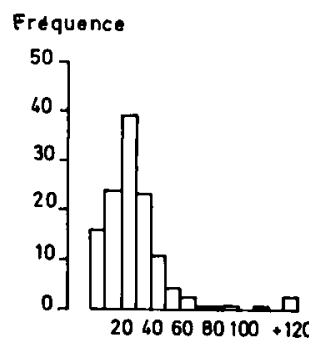

6eme semaine

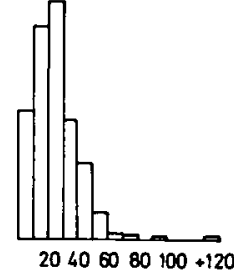

Intervalles en minutes
(B)

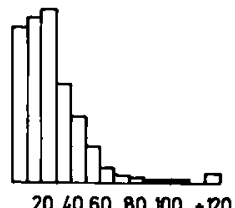

lo eme semaine

FIG. 2. -- Polygones de fréquence des intervalles entre prises d'aliment successives au cours des sixième, douzième et dix-huitième semaines et pendant les périodes d'éclairement (A) et d'obscurité (B)

Chaque polygone correspond à la fréquence moyenne des données enregistrées au cours d'une semaine sur les neuf lapins soumis au contrôle ; les intervalles d'une durée supérieure à I 20 minutes ont été regroupés au sein d'une seule classe compte tenu de leur dispersion.

Aliment

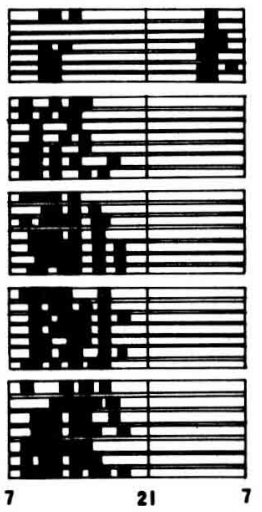

6e semaine

9e semaine

12. semaine

15e semaine

18e semaine

houre
Eau de boisson
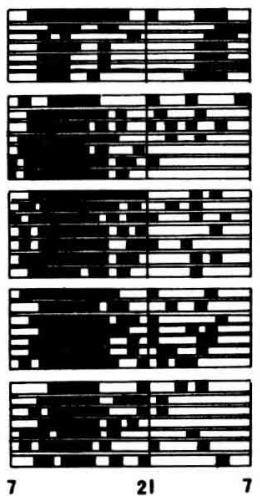

21

\section{repos alimentaire

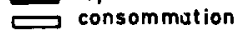

FIG. 3. - Répartition àes périodes horaires de repos ct d'activité alimentaire chez l'un des lapins expérimentaux au cours des sixième, neuvième, douzième, quinzième et dix-huitième semaines

Le nycthémère est divisé en 24 périodes horaires, chaque période est représentée en blanc ou noir selon que l'animal y a effectué ou non des prises d'aliment ou d'eau de boisson. Chaque ligne représente une journée d'enregistrement. Le trait vertical marque pour chaque journée la fin de la période d'éclairement et le début de la phase d'obscurité. 
contrôlés, très représentatif de l'ensemble des 9 lapins étudiés, il existe dès la sixième semaine un repos alimentaire diurne, apparaissant à la même heure d'un jour à l'autre et d'une durée qui a tendance à augmenter avec l'âge pour atteindre parfois 5 à 6 heures consécutives en ce qui concerne l'aliment et 8 à 9 heures pour la boisson. Chez le jeune de 6 semaines, il existe le plus souvent la nuit une période de repos alimentaire qui disparaît avant la neuvième semaine.

L'examen des histogrammes de la figure 2 permet encore de remarquer l'augmentation avec l'âge de la fréquence absolue et relative des intervalles de longue durée entre prises au cours de la période d'éclairement, le phénomène étant inversé pendant la période d'obscurité. Autrement dit en passant de 6 à I 2 puis I 8 semaines, le I apin semble espacer ses prises d'aliment diurnes et répéter plus fréquemment ses repas nocturnes; l'étude de ce point sera complétée au paragraphe C.

\section{B. - Aspects quantitatifs de la consommation d'aliment et d'eau}

Au tableau I sont reportées les valeurs moyennes \pm écart-type et les coefficients de variation des quantités, fréquences et poids moyen des prises d'aliment et d'eau par jour ainsi que le poids des sujets expérimentaux.

TABLEAU I

Principales caractéristiques de la consommation moyenne journalière d'aliment et d'eau de boisson de neuf lapins mâles en croissance

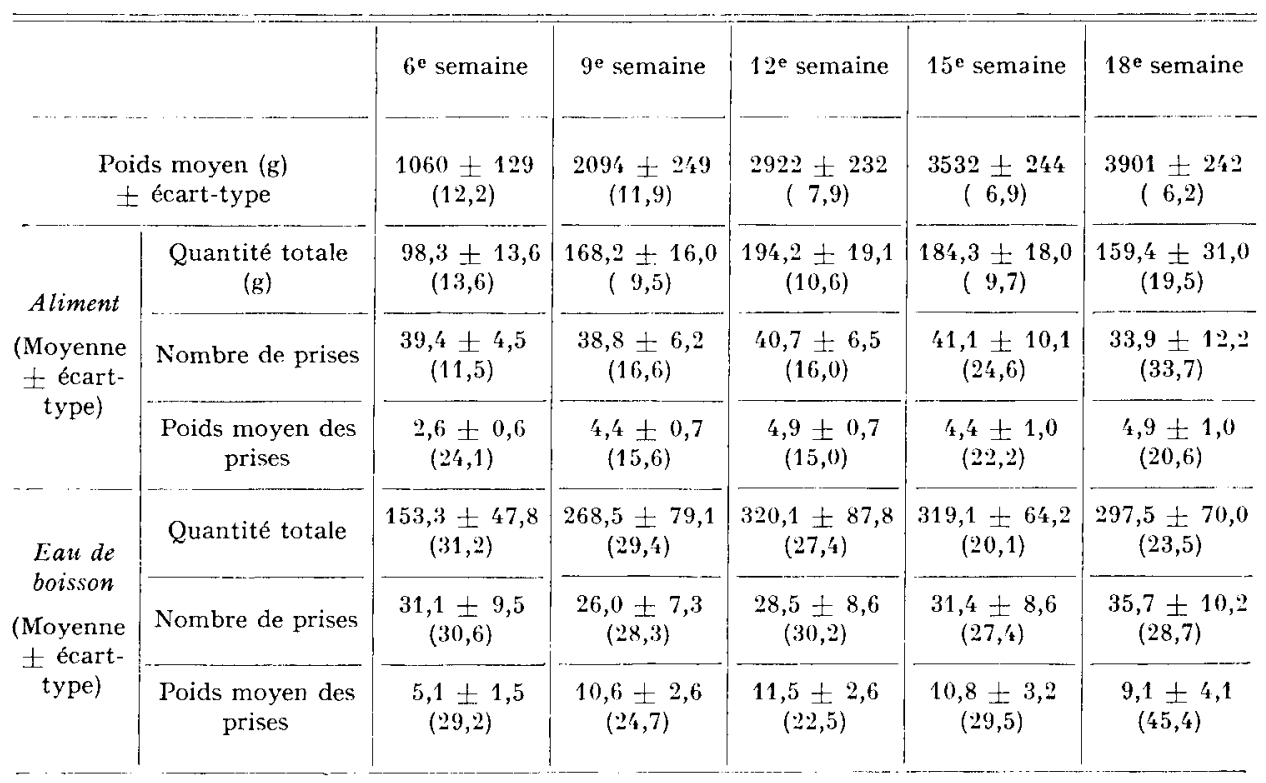

* Entre parentheses figurent les coefficients de variation

$$
\text { c.v. }=\frac{\text { écart-type }}{\text { moyenne }} \times 100
$$


Au tableau 2 figurent les valeurs des mêmes paramètres au cours des périodes de lumière et d'obscurité.

\section{TABLEAU 2}

livolution au cours de la croissance des caractéristiques de la consommation d'aliments de neuf lapins pendant les périodes d'éclairement et d'obscurité

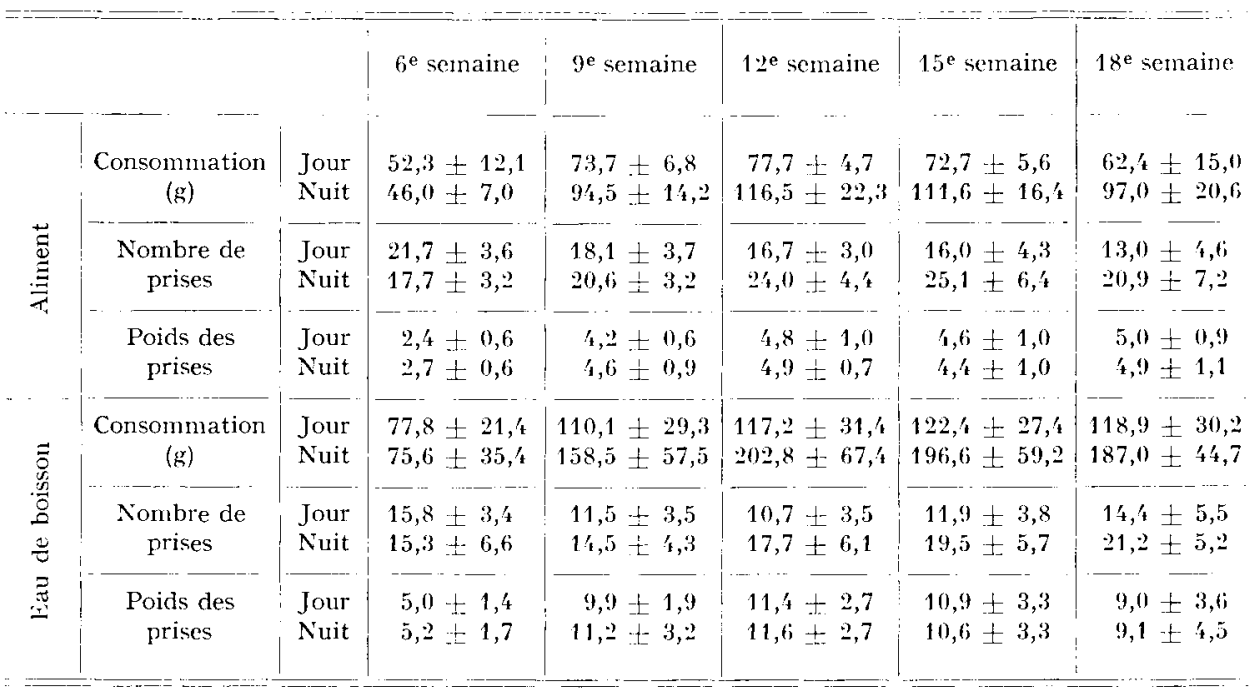

- Croissance : la croissance des sujets expérimentaux est rapide ; 1'homogénéité du lot augmente avec l'âge (coefficient de variation s'abaissant de 12,2 à 6 semaines jusqu'à 6,2 à $I 8$ semaines).

- Consommations d'aliment et d'eau : elles augmentent significativement de la sixième à la douzième semaine ; elles tendent à diminuer à la dix-huitième. Ces variations de niveau d'ingestion sont presque entièrement dues aux modifications de la consommation nocturne (tabl. 2) la consommation diurne variant peu. Nous avons comparé intra-individu, à l'aide du test F, les consommations diurnes, nocturnes et journalières enregistrées aux neuvième, douzième, quinzième et dix-huitième semaines; seules les différences de consommation nocturnes et journalières présentent des variations hautement significatives $(P<0,0 I)$ les consommations diurnes ne diffèrent pas d'une semaine à l'autre.

Le rapport eau de boisson/aliment augmente avec l'âge de $I, 55$ à I,87 ce qui représente des rapports eau/matière sèche allant de $I, 86$ à 2,22 .

Il faut souligner la très forte variabilité des quantités d'eau consommées quel que soit l'âge, en dépit d'une température maintenue constante, mais en l'absence d'une régulation de l'humidité relative. La consommation d'aliment est moins variable si l'on excepte la dix-huitième semaine où la variabilité devient forte.

- Fréquence des prises : globalement le nombre moyen quotidien des prises est relativement constant jusqu'à la quinzième semaine puis il diminue (aliment) ou augmente (boisson) à la dix-huitième semaine. Une analyse individu par individu 
fait apparaître des tendances très différentes de l'un à l'autre si 1'on excepte la dixhuitième semaine où se généralise la diminution de fréquence des prises d'aliment.

L'analyse séparée des consommations diurnes et nocturnes (tabl. 2) met en lumière l'existence d'une évolution de la fréquence des prises avec 1'âge : tendance à la diminution de fréquence des prises diurnes d'aliment alors que les prises nocturnes ont une fréquence accrue jusqu'à la quinzième semaine. En ce qui concerne la boisson le nombre de prises diurnes ne varie pas de façon significative alors que la fréquence des prises nocturnes augmente.

- Amplitude des repas : des tableaux I et 2 il ressort :

- les quantités d'eau et d'aliment consommées par prise, après avoir augmenté entre 6 et 9 semaines, varient peu au-delà ;

- il n'y a pas de différence d'amplitude moyenne des prises diurnes et nocturnes quel que soit 1'âge ;

- 1'amplitude des prises est extrêmement variable; ainsi, à 6 semaines les prises d'aliment et de boisson varient respectivement de 0,25 à $8 \mathrm{~g}$ et de 0,5 à $\mathrm{I} 6 \mathrm{~g}$; à partir de 9 semaines les fluctuations sont encore plus importantes : 0,25 à I 6 g pour 1'aliment, 0,5 à $40 \mathrm{~g}$ pour la boisson.

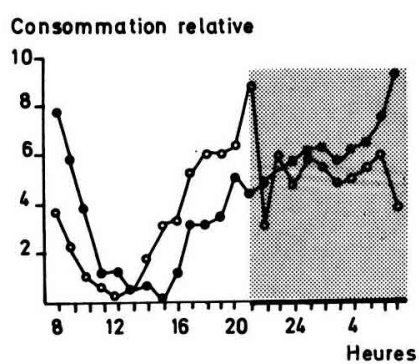

Frequence relative

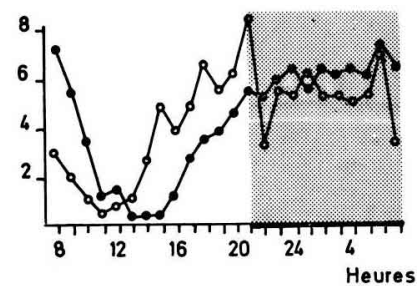

(B)

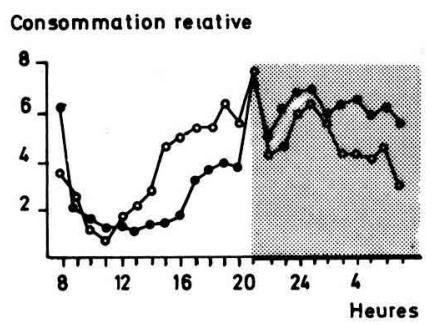

Frequence relative
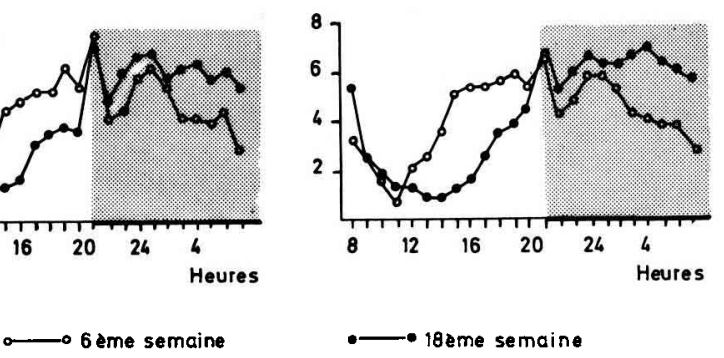

$\bullet-\rightarrow$ reme semaine

FIG. 4. - Fréquence relative des repas

et consommation relative d'eau (A) et d'aliment (B)

au cours du nycthémère chez des lapins âgés de 6 et 18 semaines

Les consommations relatives et les fréquences relatives sont exprimées en p. Ioo, de la consommation moyenne quotidienne et du nombre de prises moyen quotidien.

La zone ombrée correspond à la période d'obscurité 


\section{C. - Répartition nycthémérale de la consommation}

Compte tenu de l'évolution du caractère " nocturne " du lapin au cours de la croissance nous avons représenté, en les rapportant à la même échelle (indice roo en 24 heures), les consommations moyennes horaires d'aliment et d'eau des lapins à l'âge de 6 et de 18 semaines ainsi que les fréquences moyennes horaires correspondantes.

On notera (fig. 4) la très grande analogie des courbes représentant la fréquence des prises et la quantité consommée ce qui résulte de l'absence de variations nycthémérales d'amplitude des repas.

En ce qui concerne la consommation d'aliment solide, le niveau d'ingestion s'abaisse au cours des 3 ou 4 premières heures de lumière puis s'élève à nouveau dès la cinquième heure chez les sujets de 6 semaines, après un délai plus long chez les lapins de $\mathrm{I} 8$ semaines. La consommation d'aliment passe par un maximum, au cours de l'heure qui précède l'extinction des lumières, suivi immédiatement d'une dépression. Pendant la phase nocturne, le niveau moyen relatif de consommation du sujet de I8 semaines est toujours plus élevé que chez le jeune.

En ce qui concerne la boisson, l'allure générale de la consommation est, à 6 semaines, très comparable à celle de l'aliment. A dix-huit semaines, par contre, le Lapin ne présente pas de " pic " au cours de l'heure précédant l'extinction des feux ; la consommation augmente jusqu'à la fin de la nuit puis décroît pour être minimale en milieu de période d'éclairement.

\section{DISCUSSION ET CONCLUSIONS}

Cette étude a tout d'abord confirmé les caractéristiques générales du comportement spontané de prise d'aliment chez le Lapin, décrites tout d'abord chez les adultes des deux sexes (PRUD'HON et al., I972) puis sur un effectif réduit de mâles Néo-Zélandais enregistrés de 6 à 27 semaines (PRUd'hon, CARLEs et Goussopoulos, I973). Contrairement à ce qui se passe dans d'autres espèces utilisées pour l'étude des mécanismes régulateurs de l'appétit telles que le Rat blanc (LE MAGNEN et TALLON, I966) où l'on peut définir des repas, formés de prises répétées, séparés par des intervalles de longue durée, chez le Lapin, comme d'ailleurs chez le Poussin et le Poulet (Goussopoulos et al., I973 b), les prises sont espacées de telle façon qu'on ne peut faire apparaître de repas nettement individualisés.

Cette étude nous a apporté des renseignements entièrement nouveaux sur certaines étapes de l'adaptation du jeune lapereau à une alimentation solide et non plus lactée et sur 1'accentuation progressive de l'aspect " nocturne » du comportement alimentaire.

Il est tout à fait remarquable qu'un jeune qui jusqu'à 4 semaines a tété presque exclusivement le lait maternel, en fin de nuit et en une seule fois (ZARRow et al., I965), soit capable une semaine plus tard de réaliser une quarantaine de prises solides et une trentaine de prises liquides réparties sur tout le nycthémère, avec un maximum de consommation situé immédiatement avant le début de la nuit ; cela dénote d'exceptionnelles facultés d'adaptation que nous avions déjà soulignées dans un travail antérieur (PRUd'hoN et BFI, I968). 
Le jeune lapereau, au cours de sa sixième semaine, manifeste déjà une tendance nocturne prédominante masquée par l'existence d'un pic très marqué de consommation au cours de la dernière heure de lumière qui représente une anticipation de l'extinction des feux - phénomène que nous avons déjà décrit chez le Poulet (Goussopoulos et al., I973a) - et chez le Lapin (Prud'Hon, Caries, GoussoPoulos, I973). A cet âge toutefois il manifeste encore deux phases de repos alimentaire l'une diurne qui va subsister et s'étendre, l'autre nocturne qui disparaîtra. L'accentuation de l'aspect nocturne des consommations, qui se traduit par un espacement des repas diurnes et une augmentation de fréquence des prises nocturnes n'avait pas été décrit à notre connaissance chez le Lapin. Dans le cas du Rat blanc qui présente à peu près la même proportion de consommation nocturne que le Lapin (60 p. IOO environ) LE MAGNEN (I970) a émis l'hypothèse de l'existence d'un cycle métabolique journalier comportant une insulino-sécrétion élevée la nuit stimulant une lipogénèse active et un haut niveau d'ingestion; le jour par contre une importante sécrétion d'adrénaline mobiliserait les acides gras provoquant un abaissement du niveau de consommation. L'étude des relations entre lumière et consommation est poursuivie. Il a été démontré notamment qu'un décalage de la période de distribution de la lumière entraînait, en moins de ${ }_{5} 5$ jours, une réadaptation du comportement du lapin à son nouveau régime lumineux (CHERUBIN, I974).

Fin ce qui concerne l'aspect quantitatif de la consommation d'aliments, nous avons enregistré un niveau d'ingestion sensiblement plus élevé que lors de nos essais de I967, réalisés il est vrai sur des sujets d'une autre race, dans un clapier non conditionné thermiquement et avec un aliment granulé beaucoup plus riche en matières protéiques. Or des travaux récents (Arkhurst, I973, Colin, Arkhurst et LEBAS, I973) laissent penser que la nature des acides aminés de l'aliment pourrait avoir une influence sur le niveau d'ingestion notamment dans le cas d'un régime riche en méthionine qui entraînerait une baisse de celui-ci. I,a diminution de consommation enregistrée au cours de la dix-huitième semaine ne semble pas anormale chez des sujets ayant eu une croissance très rapide, arrivés au voisinage de leur poids adulte.

Enfin sot1lignons que le rapport entre l'ingestion d'eau et de matière sèche est proche de 2 et tend à augmenter avec l'âge ; ces résultats confirment ceux de PRUD'HoN (1967) et CizEK (I96I) qui avaient déjà signalé une évolution du rapport eau/matière sèche avec 1'âge. Selon LE MaGNEN, le Rat blanc établit un rapport constant de sa prise hydrique à sa prise solide, spécifique de l'aliment qu'il reçoit.

L'étude des répercussions d'une restriction hydrique sur le comportement alimentaire du lapin et des conséquences zootechniques qui en découlent a été entreprise.

Reçu pour publication en décembre 1974.

\section{SUMMARY}

\section{CHANGE IN THE SOLID AND LIQUID FOOD INTAKE CHARACTERISTICS DURING GROWTH OF AD LIBITUM FED DOMESTIC RABBITS}

The amount and distribution of solid and liquid food intake were recorded graphically in nine New Zealand male rabbits during the course of the 6 th, 9 th, 12 th, I 5 th and 18 th weeks of age. The recording device was described by PRUD'HON, CARLES and Goussopoulos (I973). 
'The feeding behaviour of the growing rabbits was characterized by the following facts :

- absence of real meals, but a succession of food and water intakes of very irregular patterns concerning both amounts and intervals.

- circadian distribution of intake, closely related to the presence or absence of light.

-- progressive increase of nocturnal feeding habits.

Intake of food and water increased up to the 15 th week of age and decreased at the 18 th weck. Between the 6th and 9 th weeks after birth, this increase was due to greater number of nocturnal meals varying only slightly in volume. Simultaneously, the frequency of diurnal meals tended to decrease. The mean volume of both diurnal and nocturnal meals was the same whatever the age of the animals. The water/DM ratio increased with age (from 1.86 to 2.22).

The circadian distribution of feeding was characterized by :

One period of low diurnal feeding activity of short duration (3 hours) during the 6th week of age and one of longer duration (5-7 hours) during the i 8 th week of age.

In addition, at the 6 th week of age, there was a period of lower food intake during nights, but not so in older animals.

A peak was observed in the fecling activity within the hour preceding the switching off the light. The water intake presented this peak in animals of 6 wecks of age, but not in those of 18 weeks. At this age, feeding activity was maximum at the end of the night.

\section{RÉFÉRENCES BIBLIOGRAPHIQUES}

Arkhurst C., I973. Effet chez le Lapin en croissance de l'addition de DL-méthionine au régime alimentaire. Thèse Dr spécialité Paris VI.

CherUbrx M., I974. Étude du comportement alimentaire du lapin domestique, du sevrage à la dix-huitième semaine. Influence de quelques facteurs extrinsèques sur les paramètres de l'ingestion alimentaire. Thèse I)r spécialité. Université des Sciences et Techniques du Languedoc, Montpellier.

Cr\%k L. J., I96r. Relationship between food and water ingestion in the rabbit. Am. J. Physiol., 201, $557-566$.

Colin M., Arkhurst G., Lebas F, i973. Fffets de l'addition de méthionine au régime alimentaire sur les performances de croissance chez le Lapin. Ann. Zootech., 22, 485-491.

Gorssopollos J., Carles Y., Prud'hon M., Bacou F., I973a. Enregistrement graphique de l'activité et du comportement alimentaire du Poulet. Ann. Zootech., 22, I33-I 45.

Goussopollos J., Carles Y,, Prud'hon M., I973 b. Comportement alimentaire du Poussin, de la naissance à 3 semaines. J. Rech avic. et cunicoles. I. N. R. A., ITAVI, B4 I5r-I58. ITAVI, Paris edit.

LE MACiNex J, I970, in Klötz H. J., Les hormones et le comportement. L'expansion. Paris 7-28.

Le Magnen J., Talion S., ig66. Ja périodicité spontanée de la prise d'aliments ad libitum du Rat blanc. J. Physiol. Paris, 58, 328-349.

PrUd'hox M., г967. L'appétit du lapin alimenté à sec. Bull. Tech. inf. ingrs, servs, agric, 219, I-I6.

Prud'hos M., Bel L., I968. Le sevrage précoce des lapereaux et la reproduction des lapines. Ann. Zootech., 17, 23-30.

Prtidnon M., Carles Y., Goussopollos J., Kokhl P. F., 1972. Enregistrement graphique des consonmations d'aliments solide et liquide du lapin domestique nourri ad libitum. Ann. Zootech., $21,45^{1-460 .}$

Priv'hon M., I973. Le comportement alimentaire du lapin en croissance. Mise au point d'une méthode d'étude et premières observations. Con ${ }^{r}$. Int. Côme. Sept 197.3, 3 I $^{-325}$, Camer a di Commercio industria antigianato et agricoltora, LRBA.

JarroW M. X., DinenberG I. M., Axpresos C. O., 1965. Rabbit frequency of suckling in the pup. Science, 150, 1835-36. 